\title{
Fast Power and Channel Adaptation for Mobile Users in OFDMA Multi-Cell Scenarios
}

\author{
L. Reggiani ${ }^{1}$, L. Galati Giordano ${ }^{2}$ and L. Dossi ${ }^{3}$ \\ ${ }^{1}$ Politecnico di Milano \\ 2,3 IEIIT CNR - Milano \\ Italy
}

\section{Introduction}

Methods for interference mitigation and adaptive multi-user resource allocation are among the most promising technological breakthroughs that should improve capacity of the last generation broadband wireless systems. In multi-cell scenarios characterized by radio technologies based on OFDMA (Orthogonal Frequency Division Multiple Access), co-channel interference is the performance limiting factor and techniques that reduce transmission power, act indirectly on the co-channel interference levels. In this work we investigate multi-cell scenarios with mobile users in which power and channel adaptation act as positive factors on the interference reduction. We are interested particularly on the role of closed loop power control and of simple techniques for fast channel assignment in multi-cell configurations with low reuse factors (possibly between 1 and 3). From the numerical findings presented in this chapter, it turns out that spectral efficiency can be improved by algorithms that are simple and fast enough to be exploited for mobile users, at least at pedestrian velocities.

The ambitious capacity requirements for future broadband wireless networks expose system designers to the challenging compromise between the scarcity of spectral resources and the impairments introduced by radio propagation randomness. At the heart of this challenge there is the ability to exploit radio resources as efficiently as possible in all available dimensions (space, time, frequency or channel, power, modulation and coding). Since mitigation of interference and resource allocation strategies are usually studied separately, in this work we aim to investigate more deeply the relations between the application of simple techniques for allocating power and channels to mobile users and their impact on interference and, consequently, on multi-cell overall performance.

Adaptive allocation techniques have already demonstrated their ability to mitigate the effect of deep fading by encouraging channel access to users temporarily experiencing better propagation conditions, taking advantage by the so-called 'multiuser diversity gain' (Knop \& Humblet (1995)). In our previous works (Galati et al. (2010); Reggiani et al. (2007); Galati et al. (2008)) we have shown how resource allocation algorithms in multi-cell environments have a non-trivial impact on downlink mutual interference among cells when the simple mechanism of power adaptation is applied to the system sub-channels: when the power, after channel assignment, is reduced to the minimum level required for the selected modulation and coding profile, all the network acquires an advantage from the interference reduction, giving rise to a 'virtuous loop' in which performance parameters as spectral efficiency or coverage turn out 
to be improved significantly. So the decrease of transmitted power levels, possibly due to the efficient use of resources, reveals an interference mitigation impact even if the algorithms or the allocation strategies are not directly designed or applied for this final effect. More precisely, in (Reggiani et al. (2007)) different specific adaptive allocation algorithms were applied, on a cell-by-cell basis, to a multi-cell system with fixed terminals and air interface parameters compliant to IEEE 802.16 (WiMax) AMC standard (IEEE Std 802.16 (2004); IEEE Std 802.16e (2005)).

In this chapter, we overcome the fixed users assumption of our previous works and we examine a downlink/uplink scenario with mobile users focused on OFDMA physical layer architectures as in the recent LTE (Long Term Evolution) standard and in the previous IEEE 802.16. In particular, we discuss the performance outcomes considering, at the same time, the updating rate of the algorithms, which is clearly one of the main constraints for their application to mobile users. As already mentioned, we examine two relevant issues: (i) the role of fast, closed loop, power adaptation in the multi-cell interference mitigation and (ii) the positive impact of very fast and simple allocation techniques, well suited to the application within a mobile scenario. The key point under the analysis of these techniques is the power reduction that is made possible either as a response to channel variations (power control) or as effect of advantageous channel assignments. The final, positive impact is a reduction of the overall network interference. So the novel contributions reported in this work are (i) the evaluation of allocation techniques and fast loop power adaptation on the multi-cell interference scenario with mobile users and (ii) the principle of set partitioning applied to allocation strategies for enhancing the response rate of these techniques (Sect. 3).

The chapter is organized as follows: Sect. 2 describes the system model and the channel assumptions, Sect. 3 reports the proposed fast adaptive strategies and Sect. 4 resumes the assumptions and procedures adopted in the analysis and in the simulations. Finally Sect. 5 illustrates the numerical results in terms of spectral efficiency, allocation updating rate and complexity reduction.

\section{System model}

The simulation scenario is a network of cell sites, each one divided into three hexagonal sectors and with a frequency reuse factor equal to 3 (Fig. 1). Performance is simulated only for users belonging to the center sector; the surrounding base stations (BS) generate two tiers of interferers. Base stations are equipped with directional antennas, whose patterns are taken from the 3GPP standard document (3GPP TR 25.996 (2003)) while mobile terminals are equipped with omnidirectional antennas. The frequency selective time variant channel model is derived from (3GPP ETSI TR 101112 (1998)), which is dedicated to test environments and deployment models. We select one vehicular model with a power delay profile of type $V e h-A$, corresponding to a delay spread of $370 \mathrm{~ns}$ and two pedestrian models of type Ped $-A$ and $P e d-B$, characterized by delay spreads of $45 \mathrm{~ns}$ and $750 \mathrm{~ns}$ respectively. All the models are described by a power delay profile with 6 or 4 taps at fixed delays and a classic Doppler spectrum whose bandwidth depends on the user velocity $v$. Antenna and channel parameters are summarized in Table 1, the tapped-delay line parameters of vehicular and pedestrian test environments in Table 2.

Our case study is a scalable-OFDMA (S-OFDMA) system, in which the total available bandwidth is divided in $N_{s c}$ sub-carriers, each one associated to a bandwidth that is much smaller than the coherent channel bandwidth but greater than the inverse of the channel coherence time. Then sub-carriers are grouped into $N_{S}$ sub-channels and a user is assigned 


\begin{tabular}{|l|c|}
\hline \multicolumn{2}{|c|}{ Base station equipment } \\
\hline Antenna Gain & $17 \mathrm{dBi}$ \\
\hline 3-dB beamwidth & $70^{\circ}$ \\
\hline Maximum BS power & $35 \mathrm{dBm}$ \\
\hline \hline \multicolumn{2}{|c|}{ User station equipment } \\
\hline Antenna Gain & $0 \mathrm{dBi}$ \\
\hline Radiation Pattern & Omnidirectional \\
\hline Noise figure Channel model \\
\hline \hline \multicolumn{2}{|c|}{ Ch dB } \\
\hline Path loss & $P L(d)=136.7+39.7 l_{0} g_{10}(d)$ \\
& {$[P L$ in dB; $d$ in km] } \\
\hline Center frequency & $3.5 \mathrm{GHz}$ \\
\hline Fast fading & Veh-A, Ped-A or Ped-B model \\
\hline
\end{tabular}

Table 1. Antenna, channel model and equipment parameters.

one or more sub-channels for its traffic. The number $N_{U}$ of users admitted to the service will be smaller or equal to $N_{S}$. A sub-channel can be constituted by adjacent or distributed sub-carriers. In our simulations, we will consider adjacent sub-carrier allocation modes (named as 'AMC' in IEEE 802.16 standard). For example, in case of AMC permutation zone, each sub-channel consists of a group of 18 contiguous sub-carriers (16 data and 2 pilots) where fading is assumed correlated. With a bandwidth of $10 \mathrm{MHz}$ per sector and an FFT (Fast Fourier Transform) size equal to 1024 , the sub-carrier spacing $\Delta f$ is $11.16 \mathrm{kHz}$ and the number of sub-channels per sector is $N_{S}=48$.

As the fading is assumed flat in each sub-channel, the instantaneous channel state indicator (CSI) of the $i$-th sub-channel, seen by the $k$-th user, is defined as

$$
\beta_{k, i}=\frac{P_{N}+I_{k}}{H_{k, i}}
$$

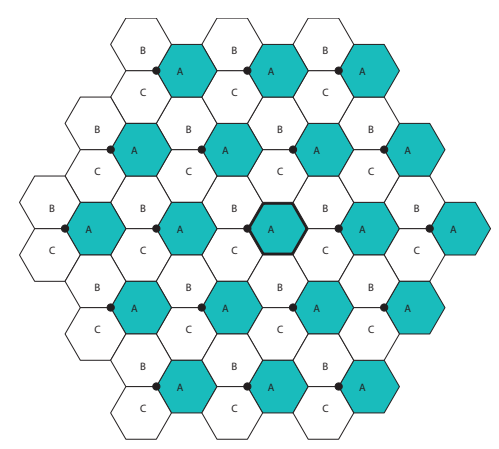

Fig. 1. Network topology: the sectors identified by the same letter (A, B or C) employ the same frequency band. Circles denote the base stations and the center sector is delimited by a bold line. 


\begin{tabular}{|c|c|c|}
\hline \multicolumn{3}{|c|}{ Veh-A Model } \\
\hline Tap & Rel. Delay (ns) & Avg. Power (dB) \\
\hline 1 & $\overline{0}$ & 0.0 \\
\hline 2 & 310 & -1.0 \\
\hline 3 & 710 & -9.0 \\
\hline 4 & 1090 & -10.0 \\
\hline 5 & 1730 & -15.0 \\
\hline 6 & 2510 & -20.0 \\
\hline \multicolumn{3}{|c|}{ Ped-A Model } \\
\hline Tap & Rel.Delay (ns) & Avg. Power (dB) \\
\hline 1 & 0 & 0.0 \\
\hline 2 & 110 & -9.7 \\
\hline 3 & 190 & -19.2 \\
\hline 4 & 410 & -22.8 \\
\hline \multicolumn{3}{|c|}{ Ped-B Model } \\
\hline Tap & Rel.Delay (ns) & Avg. Power $(\mathrm{dB})$ \\
\hline 1 & 0 & 0.0 \\
\hline 2 & 200 & -0.9 \\
\hline 3 & 800 & -4.9 \\
\hline 4 & 1200 & -8.0 \\
\hline 5 & 2300 & -7.8 \\
\hline 6 & 3700 & -23.9 \\
\hline
\end{tabular}

Table 2. Test Environment Tapped-Delay-Line Parameters

where $H_{k, i}$ is the channel gain, including path loss, fast fading and antenna gains, $P_{N}$ is the noise power and $I_{k}$ is the instantaneous interference power generated by the surrounding co-channel interferers. We do not generally assume the ideal instantaneous estimation of $I_{k}$ in Equation 1 but only of an average interference power $\bar{I}_{k}$ since random fluctuations of $I_{k}$ appear unpredictable without a distributed control among the interfering BSs. Moreover, as we are considering mobile terminals, imperfect estimation of channel gains $H_{k, i}$ can derive from the fact that the algorithm updating time (i.e. the interval between two consecutive applications of the allocation algorithm) is not negligible w.r.t. the channel coherence time. Assuming that only an average interference power $\bar{I}_{k}$ can be known at the algorithm processing unit (typically at the BS), the interference variations, not perfectly estimated, are compensated by an interference (and noise) margin $F M_{I}$, while the variations experienced by the channel gain, due to Doppler effect, are compensated by a Doppler margin $\triangle F F$, resulting in the new CSI definition

$$
\gamma_{k, i}=\Delta F F \cdot F M_{I} \frac{P_{N}+\bar{I}_{k}}{H_{k, i}}
$$

which is the general input of the allocation algorithms. The two functions 1 and 2 are directly related to the signal-to-interference noise ratio (SINR) at the $i$-th sub-channel for the $k$-th user by means of the transmitted power $P_{k, i}$.

If we consider, for each modulation and coding profile $l=1 . . L$, the number $c_{l}$ of information bits provided per carrier and the lower SINR bound $\alpha_{l}$ for its correct functioning, each sub-channel $i$ is supposed to transmit successfully $c_{k, i}=c_{l}$ information bits per carrier for the $k$-th user if the algorithm is able to assign a power $P_{k, i}: S I N R_{k, i}=P_{k, i} / \gamma_{k, i}=\alpha_{l}$. In 
our simulations, the thresholds $\alpha_{l}(1 \leq l \leq L)$ are assumed equally spaced between the minimum and the maximum values specified in modulation and coding profiles for IEEE 802.16 S-OFDMA standard $\left(\alpha_{1}=2.88 d B\right.$ and $\left.\alpha_{L}=17.50 d B\right)$. For the sake of generality, instead of considering a number $c_{l}$ of information bits provided per carrier that depends on the chosen modulation and coding, we assume that an user, whose SINR achieves the threshold $\alpha_{l}$, transmits with the theoretical Shannon efficiency

$$
\eta_{l}[\mathrm{bit} / \mathrm{s} / \mathrm{Hz}]=\log _{2}\left(1+10^{\alpha_{l} / 10}\right) .
$$

If none of the thresholds is exceeded, sub-channel $i$ is switched off for the $k$-th user.

\section{Fast adaptive techniques}

In OFDMA systems, smart allocation of radio resources is a crucial aspect for achieving excellent performance levels. In Fig. 2 we can observe a typical structure of an OFDMA time-frequency layer: the set of sub-carriers and symbol times is divided into resource blocks (RB), which constitute the minimum amount of resources that can be assigned to an user connection. In fact each user is assigned a set of RBs, generally but not necessarily contiguous (Fig. 2(a)). The sub-carriers of the same RB are interested by the same modulation, coding profile and power. For the sake of simplicity but without loss of generality w.r.t. the scope of this study, we assume a resource division based on a one-dimensional approach, where a user is assigned the same sets of sub-carriers for the entire allocation time $T_{U P D}$ (Fig. 2(b)). So a resource block is equivalent to a sub-channel and an user can share separate sub-channels in the same $T_{U P D}$.

The allocation techniques require knowledge of the function reported in Equation 1 with an updating time $T_{U P D}$ that should be shorter than the coherence time of the channel; this constitutes the main limiting factor in mobile applications. When $T_{U P D}$ is comparable or greater than the coherence time of the channel, the algorithm performance degrades rapidly as the channel gain in that updating interval can experience heavy fluctuations due to the Doppler effect. In order to compensate this degradation, our simulations pre-compute the 'Doppler margin' $\triangle F F$ in Equation 2 as the channel gain variation that each sub-channel exceeds, during an entire period $T_{U P D}$, with a probability equal to 0.10 . So $\triangle F F$ is used in the new CSI estimate as in Equation 2. As expected, this Doppler margin depends both on the updating time and on the channel coherence time, which is function of the mobile user velocity $v$.

In the sequel, we present a study on the impact of fast closed loop power control on interference (Sect. 3.1) and of techniques for exploiting multi-user diversity in channel assignments also in a mobile context (Sect. 3.2).

\subsection{Closed loop power adaptation}

In OFDMA systems, power adaptation is performed either in open or closed loop modalities. It is well known that a fast closed loop mechanism plays a crucial role in CDMA cellular systems for limiting the intra-cell interference, especially on the uplink. Here we are interested to the impact of fast power adaptation on OFDMA systems with low reuse factors as a positive contribution to limiting extra-cell interference. This is true for the uplink direction, as already observed in (Schoenen \& Qin (2009); Li et al. (2008); Tee et al. (2007)), but also for the downlink side, on which we have focused our analysis. The analytical procedure presented in Sect. 5 will take into account the multi-cell interference and it will assume ideal channel knowledge and 


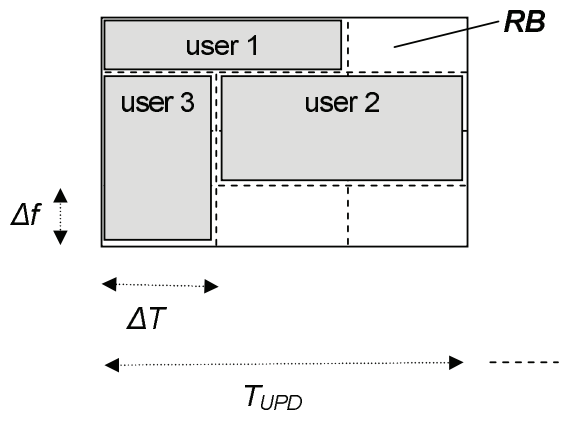

(a)

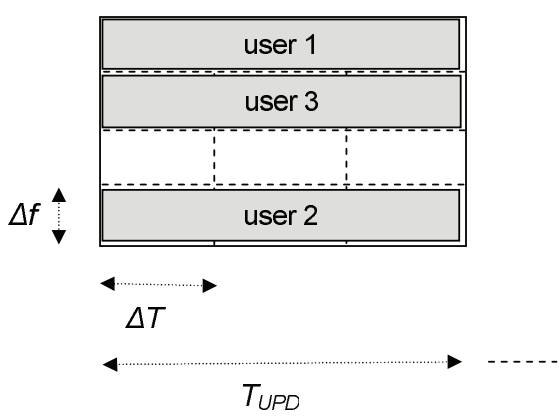

(b)

Fig. 2. Time-frequency resource organization in an OFDMA system: two-dimensional (a) and one-dimensional scenarios.

adaptation. On the other hand, the simulations will reveal the impact of these techniques for several levels of the updating time (w.r.t. the channel coherence) and with other impairments. The closed loop power adaptation (CLPA) is able to adjust the power at the base station (downlink) or at the terminal (uplink) at the level that is exactly necessary to achieve the maximum profile threshold $\alpha_{l}(1 \leq l \leq L)$ compatible with the power assigned to each sub-channel $P_{S}=P_{B S} / N_{S}$, being $P_{B S}$ the total maximum BS transmission power. In CLPA there is no allocation based on the channel state indicator but users are assigned to available sub-channels randomly. In practice, in our simulations, each user is assigned to $N_{S} / N_{U}$ sub-channels: the selected modulation profile $0 \leq l \leq L$ is given by

$$
l:\left\{\begin{array}{l}
\frac{P_{S}}{\gamma_{k, i}} \geq \alpha_{l} \\
\frac{P_{S}}{\gamma_{k, i}}<\alpha_{l+1}
\end{array}\right.
$$

where $\alpha_{0}=-\infty$, being $l=0$ associated to the absence of transmission, and $\alpha_{L+1}=+\infty$. Then power is adapted to the value

$$
P_{k, i}=\alpha_{l} \cdot \gamma_{k, i} \leq P_{S}
$$

Disequalities 4 and 5 are checked every $T_{U P D}$ seconds and either powers or modulation profiles can be changed according to the channel variations reported by the updated coefficients $\gamma_{k, i}$.

\subsection{Fast channel assignment}

As mentioned in Sect. 1, smart resource allocation in OFDMA systems is usually performed for fixed users since the assignment procedure is hardly compatible with challenging mobility constraints. The algorithm updating time is affected by the necessity of transmitting CSI at the base station (downlink) and by the computing time of the algorithm itself. Here we propose a very simple approach in which the base station does not operate on the complete group of sub-channels and users but only on small subsets. In other words, the base station does not assign a channel to a user but subsets of $N_{S} / P$ channels to $N_{U} / P$ users with $1 \leq P \leq N_{U}$ (also with small values, e.g. $N_{S} / P=3$ or 4 ) reserving one or two bits to the fast assignment communication to the users inside each subset. This solution is a partitioning procedure into $P$ sets of sub-channels and $P$ sets of users, applied to the general problem of channel assignment 
and necessary for speeding up the process (Fig. 3). Set partitioning is fixed and, by means of this procedure, the initial problem is reduced to a computational complexity that can be expressed as

$$
C_{L}=P \cdot f_{C}\left(\frac{N_{S}}{P}, \frac{N_{U}}{P}\right)<<f_{C}\left(N_{S}, N_{U}\right),
$$

with $f_{C}$ denoting a measure of the computational load of the allocation algorithm adopted in the system.

In fact, this kind of algorithm reduction to a minimum assignment problem is the way for allowing fast and light adaptation to the channel variations for mobile users. The numerical results will highlight the performance trade-offs that can be obtained at several degrees of updating time and partitioning factors. The assignment should follow the same updating time $T_{U P D}$ of the power control in Sect. 3.1; this means that, each $T_{U P D}$ seconds, the system reallocates the radio resources to the available users (not necessarily the same of the previous period $\left.T_{U P D}\right)$.

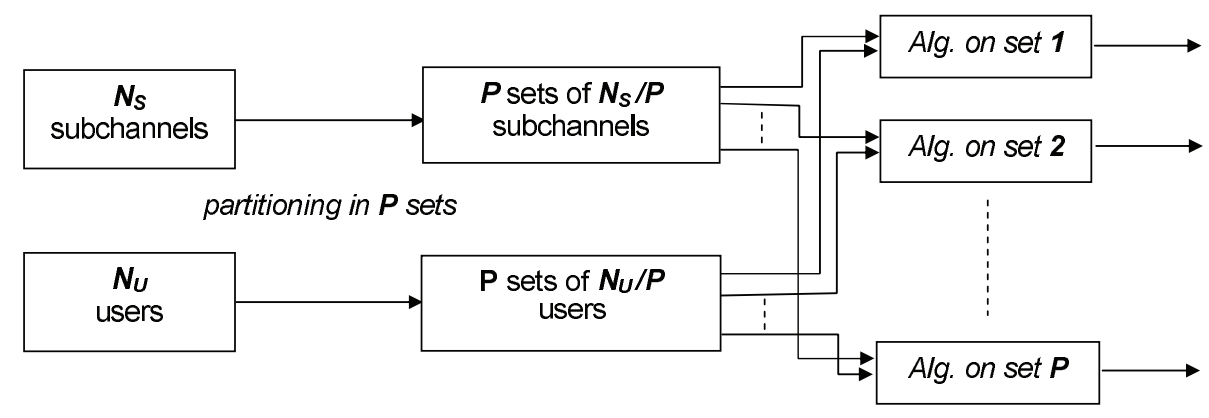

Fig. 3. Partitioned channel assignment for reduced complexity and increased speed.

\subsection{Test-bed allocation strategy}

In (Galati et al. (2008)) it is shown that, in a fading environment, the impact of a generic allocation strategy on SINR distribution can be described, in its dominant aspects, by a single parameter $I_{D}$. The use of the parameter $I_{D}$ allows us to not consider, in this study, a particular allocation algorithm, which is not our objective here (a high number of examples are present in the literature), but to focus our attention on the impact produced on the network. So we investigate the overall system by using this simple parameter and by avoiding long and useless discussions about the details of numerous solutions. In practice, when we are interested on a particular allocation solution, we can estimate its $I_{D}$ in order to have immediately a measure of its impact on network performance. As allocation algorithms usually operate with many different parameters and constraints, this $I_{D}$ assignment could require an a-posteriori estimation. In the simulations of this work and in our comparative study, we simulate a simple allocation algorithm where we can modulate a-priori the value of parameter $I_{D}$ from 1 to its maximum value, which is, for independent fading among the users, equal to the number $N_{U}$ of active users in each cell (maximum order of multi-user diversity). We remark that this choice allows to separate the numerical results of this work from a specific choice of resource allocation algorithms for both analysis and simulations. In 
this test-bed algorithm, multi-user diversity of order $I_{D}$ is provided by a strategy that assigns a sub-group of $I_{D}$ users to each sub-channel and selects, in each sub-group, the user with the best SINR.

Another rule is introduced in the assignment of users to sub-groups, in order to give each user the same number of chances to transmit. Given $N_{S}$ sub-channels, $N_{U}$ users and a diversity order $I_{D}$, with $N_{S}=k * N_{U}$ a fair rule assigns each user to $\left(N_{S} * I_{D}\right) / N_{U}=k * I_{D}$ sub-groups. The assignment slots can be structured as a matrix with $N_{S}$ rows and $I_{D}$ columns and the assignment of slots to the users is actuated filling the matrix by rows with $k$ repetitions of an ordered list of the users $U_{1} \ldots U_{N_{U}}$, as can be seen in Fig. 4(a). In the sequel this algorithm will be denoted as $T A B_{I D}$. After this operation, the power per sub-channel is adjusted according to Equation 5 .

We observe that, when $I_{D}=1$, the algorithm corresponds to the absence of any allocation strategy since the users are allocated to the sub-channels without SINR selections. So $I_{D}=1$ can be considered as the realization of the CLPA mechanism described in Sect. 3.1. Moreover, the application of the set partitioning principle on $T A B_{I D}$ (Fig. 4(b)) highlights the main impact that a complexity reduction procedure has on the algorithm effectiveness, i.e. a reduction of the multi-user diversity. As can be observed in Fig. 4(b), after set partitioning, the effective $I_{D}$ of the reduced complexity algorithm becomes

$$
I_{D, e f f}=\min \left\{I_{D}, \frac{N_{U}}{P}\right\}
$$

Our overall framework, with the algorithm options, is sketched in Fig. 5.

\begin{tabular}{l|c|c|c|}
\multicolumn{3}{c}{$\mathrm{I}_{\mathrm{D}}=3$} \\
\cline { 2 - 4 } $\mathrm{sc}_{1}$ & $\mathrm{U} 1$ & $\mathrm{U} 2$ & $\mathrm{U} 3$ \\
\cline { 2 - 4 } $\mathrm{sc}_{2}$ & $\mathrm{U} 4$ & $\mathrm{U} 1$ & $\mathrm{U} 2$ \\
\cline { 2 - 4 } $\mathrm{sc}_{3}$ & $\mathrm{U} 3$ & $\mathrm{U} 4$ & $\mathrm{U} 1$ \\
\cline { 2 - 4 } $\mathrm{sc}_{4}$ & $\mathrm{U} 2$ & $\mathrm{U} 3$ & $\mathrm{U} 4$ \\
\cline { 2 - 4 } $\mathrm{sc}_{5}$ & $\mathrm{U} 1$ & $\mathrm{U} 2$ & $\mathrm{U} 3$ \\
\cline { 2 - 4 } $\mathrm{sc}_{6}$ & $\mathrm{U} 4$ & $\mathrm{U} 1$ & $\mathrm{U} 2$ \\
\cline { 2 - 4 } $\mathrm{sc}_{7}$ & $\mathrm{U} 3$ & $\mathrm{U} 4$ & $\mathrm{U} 1$ \\
\cline { 2 - 4 } $\mathrm{sc}_{8}$ & $\mathrm{U} 2$ & $\mathrm{U} 3$ & $\mathrm{U} 4$ \\
\cline { 2 - 4 } & & &
\end{tabular}

(a)

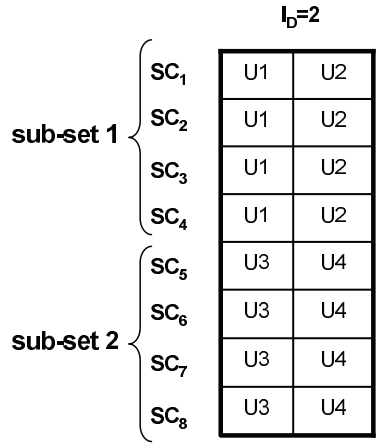

(b)

Fig. 4. Allocation of groups of users to sub-channels $s c_{i}$ with $T A B_{I D}$ strategy for $N_{U}=4$, $N_{S}=8$ and $I_{D}=3$ with $P=1$ (a) and $P=2(\mathrm{~b})$. For each sub-channel, TAB $I D$ will select the user with the best SINR in the corresponding row.

\section{Multi-cell analysis}

In multi-cell networks, SINR levels are affected by the power reduction caused by the adaptive techniques. In order to understand the behavior of the overall multi-cell system two different approaches have been implemented: (i) a completely simulative one (denoted as F1), in which the $T A B_{I D}$ algorithm runs in the simulation environment as detailed in (Reggiani et al. (2007)) and (ii) an iterative analytical approach denoted as F2 and already presented in (Galati et al. 


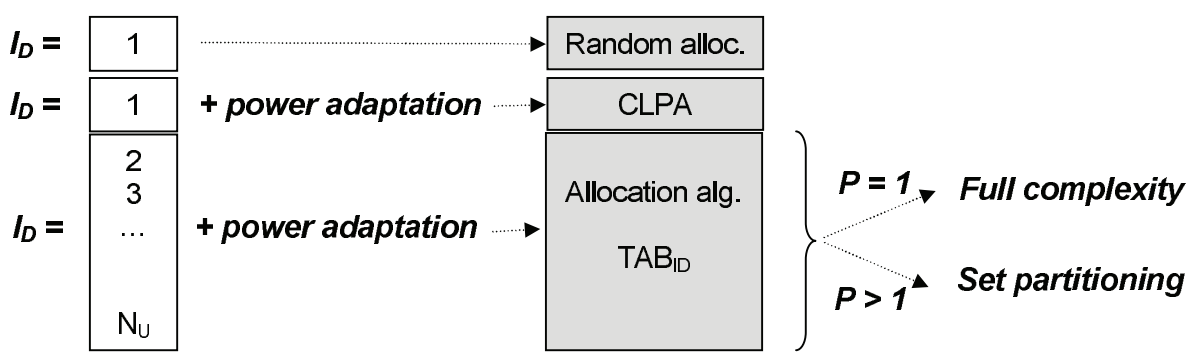

Fig. 5. Overview of the allocation options used for the numerical results.

(2008)), which reproduces the algorithm effect on SINR distribution by means of $I_{D}$, computes the power reduction and recursively applies it to the power of interfering BSs. In other words, F2 reproduces successive applications (over consecutive $T_{U P D}$ ) of a generic allocation algorithm until the system has achieved its stationary interference and SINR levels. In this work, numerical results will be focused on the final spectral efficiency for different algorithm parameters ( $P$ in Sect. 3.2, updating time), channels and user velocity (Sec. 2). The analysis is characterized by the following assumptions:

- All the active users are at distance $d$ from the six reference BS and at distance $D$ from six interfering BSs and no shadowing is present.

- Identically independent distributed (i.i.d.) fading $A_{n}$ is applied on the generic $n$ - th link ( $n=0$ for the reference link, $n=1, \ldots, 6$ for interfering links) with a probability density function $f_{A_{n}}(x)=f_{A}(x)$.

- At the first iteration $(i=0)$, the transmitted power per sub-channel in all BSs is fixed to a nominal value $P_{T X}(0)=P_{S}=P_{B S} / N_{S}$.

- At $i$-th iteration, the power reduction $\rho(i)$, which results from Equation 5 and is described by its probability density function $f_{\rho(i)}(x)$, is computed and applied to the nominal value $P_{S}$ in all the co-channel BSs, modifying their transmission power $P_{T X}(i)$.

- Channel fading is assumed non-ergodic, constant in each user transmission block.

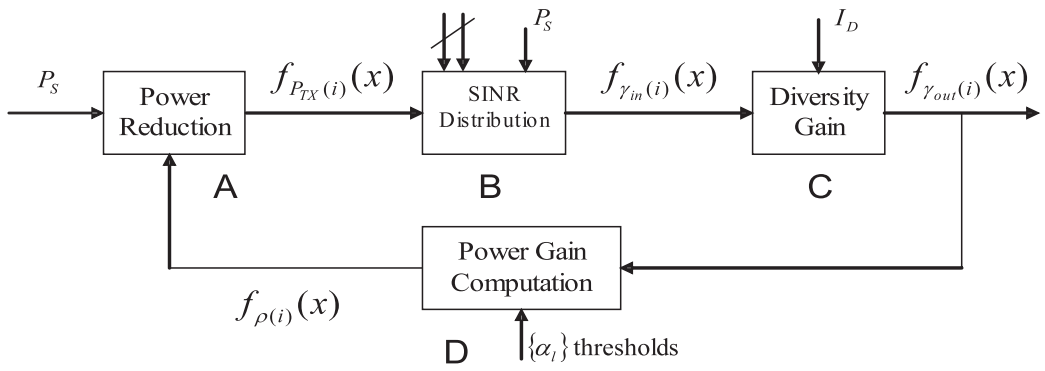

Fig. 6. Block diagram of the recursive loop for the analytical procedure $F 2$.

So, in this scenario, at $i$-th iteration, the SINR value $\gamma_{i n}(i)$ is computed in model $F 2$ as

$$
\gamma_{\text {in }}(i)=\frac{S}{I(i)+N}=\frac{P_{S} \cdot P L_{0} \cdot A_{0}}{\sum_{n=1}^{6} P_{T X, n}(i) \cdot P L_{n} \cdot A_{n}+N}
$$


where $N$, i.e. the additive white Gaussian noise power, $P L_{0}$ and $P L_{n}$, i.e. the path loss of the reference and interfering links, are deterministic parameters, while the fading $A_{0}$, $A_{n}$ and the transmission power $P_{T X, n}(i)$ in the $n$-th BS co-channel are statistical variables with probability density functions $f_{A}(x)$ and $f_{P_{T X}}(x)$ respectively. The term $I(i)$ denotes the interference at the $i-$ th iteration step. The functional block diagram of the recursive system is shown in Fig. 6: the distribution of $\gamma_{i n}(i)$ is computed in block $B$ and it is processed in block $C$ through the parameter $I_{D}$, producing the cumulative distribution function of $\gamma_{o u t}(i)$ as $F_{\gamma_{\text {out }}}(x)=\left[F_{\gamma_{\text {in }}}(x)\right]^{I_{D}}$, with $F_{\gamma}(z)=\int_{-\infty}^{z} f_{\gamma}(x) d x$. The distribution of $\gamma_{\text {out }}(i)$ goes into block $D$ that computes the distribution of power gain $\rho(i)$ (i.e. the power adaptation). Finally block $A$ closes the loop, receiving the power gain distribution $f_{\rho(i)}(x)$ and applying it to nominal transmission power $P_{S}$ of the interfering BSs. The distribution of the updated power $P_{T X}(i)$ is used for the new distribution $\gamma_{i n}(i+1)$ in the next iteration. If the initial distribution $f_{\gamma_{i n}}(x)$ cannot be derived analytically, it is obtained by simulation $(F 1)$ at the first iteration and then it is processed by $F 2$ to produce final distributions $f_{\gamma_{\text {out }}}(x)$.

\section{Numerical results}

Simulations have been performed in different configuration scenarios, mobile users at a fixed distance $d_{F I X}$ from the BS, at different distances $d$ from the BS, in the downlink or in the uplink. However some common parameters are adopted in the simulations: each BS is set to a nominal power equal to $P_{B S}=35 \mathrm{dBm}$, the number of users is fixed to $N_{U}=12$ and the number of available sub-channels is equal to $N_{S}=48$. Moreover a set of 6 SINR thresholds $\alpha_{l}$ is defined among a minimum value $\alpha_{1}=2.88 \mathrm{~dB}$ and a maximum $\alpha_{L}=17.50$ $\mathrm{dB}$. Channel fading is modelled by Veh $-A$ power delay profile for users' velocities from $v=0 \mathrm{~km} / \mathrm{h}$ to $v=60 \mathrm{~km} / \mathrm{h}$, while two different pedestrian models (Ped $-A$ and Ped $-B$ ) are used from 0 to $20 \mathrm{~km} / \mathrm{h}$. The system performance is computed and analyzed in terms of achievable spectral efficiency $\eta_{\text {out }}$ at different mobile terminals velocities, different updating times $\left(T_{U P D}=[5,10,20,40] \mathrm{ms}\right)$, in presence or not of smart radio allocation techniques and Closed Loop Power Adaptation (CLPA). The maximum spectral efficiency in the analyzed system is equal to $\max \left(\eta_{\text {out }}\right)=10 \log _{2}\left(1+10^{\left(\alpha_{L} / 10\right)}\right)=5.839[\mathrm{bit} / \mathrm{s} / \mathrm{Hz}]$.

Figs. 7-11 have been obtained in the downlink configuration. In Figs. 7-8, we show the validity of the the analytical model $F 2$ introduced in Sect. 4 w.r.t. the results obtained with intensive simulation (F1). Performance is shown in terms of the spectral efficiency $\eta_{\text {out }}$ that can be achieved using different fading models, $P e d-A$ and $P e d-B$ respectively, at different velocities of the mobile terminals (from $v=0$ to $v=15 \mathrm{~km} / \mathrm{h}$ ) and when the updating time of the allocation strategies is progressively increased, i.e. producing a new allocation configuration each $T_{U P D}=[5,10,20,40] \mathrm{ms}$. In fact, $T_{U P D}=5 \mathrm{~ms}$ means that the allocation algorithm is able to take decisions in each OFDMA frame (in IEEE 802.16 standard each frame has a duration equal to $5 \mathrm{~ms}$ ) and to distribute the available resources according to the channel state conditions (sub-channels, modulation profile and power) among the active users. Here we apply $I_{D}=N_{U}=12$, that corresponds to the configuration that is able to exploit the highest multi-user diversity order, in order to produce the maximum performance. As we can see in Fig. 7 spectral efficiency estimated by means of the $F 2$ procedure, whose results are shown with continuous lines, fits very well the values computed by means of intensive simulations $F 1$, whose results are reported with filled markers. We can notice that $\eta_{\text {out }}$ progressively decreases when $T_{U P D}$ increases as the allocation strategy loses its ability to react to the time-varying channel conditions, especially when the updating time is higher. 
Similar considerations can be done for Fig. 8, which has been derived using pedestrian channel model Ped $-B$.

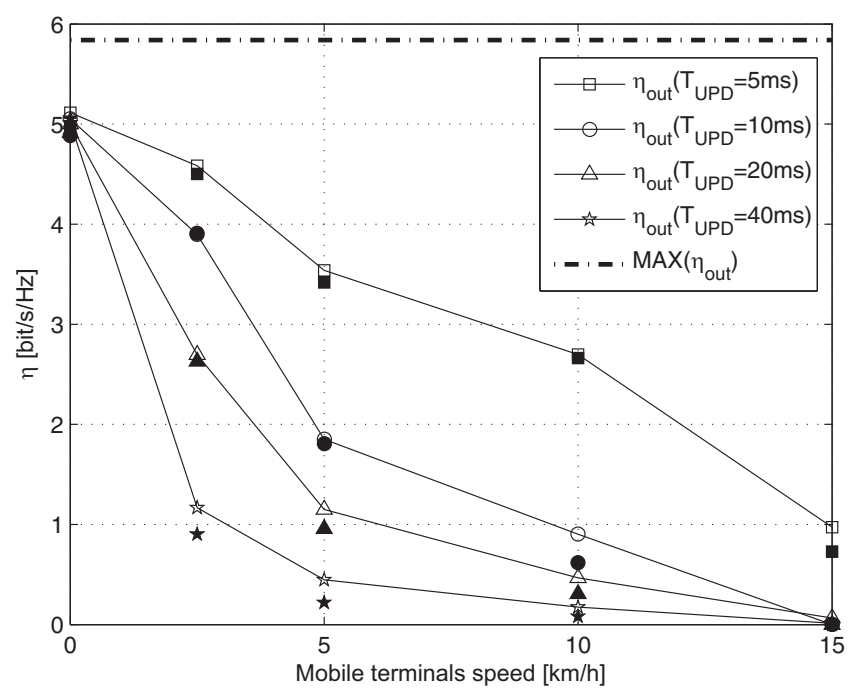

Fig. 7. Spectral efficiency $\left(\eta_{\text {out }}\right)$ as a function of user velocity $v[\mathrm{~km} / \mathrm{h}]$ with fast fading defined by pedestrian channel model $P e d-A$. Results are obtained with the analytical approach F2 (continuous lines) and compared to performance computed with intensive simulations F1 (filled markers).

Figs. 9-11 show results that are similar to those reported in Figs. 7-8 since we highlight the achievable spectral efficiency as a function of terminals velocity and algorithm updating time. However we want to stress the advantages of smart dynamic resource allocation algorithms $\left(I_{D}=N_{U}=12\right)$ over a simple mechanism of power adaptation, referred as CLPA in Sect. 3.1, which corresponds to the absence of any allocation strategies (diversity order parameter $I_{D}=$ 1). In order to have a complete comparison, we draw also the achievable spectral efficiency values when sub-channels are assigned randomly and power adaptation mechanism is not applied. This represents the worst case with $I_{D}=1$ (so absence of allocation strategy) and transmission power applied to each sub-channel always fixed to the maximum available value $P_{S}=P_{B S} / N_{S}$; it is clear the advantage provided by CLPA and particularly by even simple allocation strategies. In Fig. 9, we can observe the performance obtained with a Veh $-A$ fading model and at several velocities, from $v=0$ to $v=60 \mathrm{~km} / \mathrm{h}$. It is clear how with $v>20-30$ $\mathrm{km} / \mathrm{h}$, forming sub-channels from contiguous sub-carriers, as in the AMC configuration, is not able to react effectively to the severe channel conditions; in these cases, interference averaging strategies like mechanisms of channel permutation are more advantageous solutions (e.g. the PUSC or FUSC configurations in IEEE 802.16 standard). In fact, at high speeds, even the adoption of advanced smart allocation solutions is not effective. However, if we consider slow mobile terminals movements with average velocity within $v=15 \mathrm{~km} / \mathrm{h}$, we can notice that we achieve a considerable gain over the simple CLPA strategy when we apply radio resource allocation algorithms. For pedestrian users, Fig. 10 and Fig. 11 highlight the value of $\eta_{\text {out }}$ in the presence of $P e d-A$ and $P e d-B$ power delay profiles respectively. We can notice that, with 


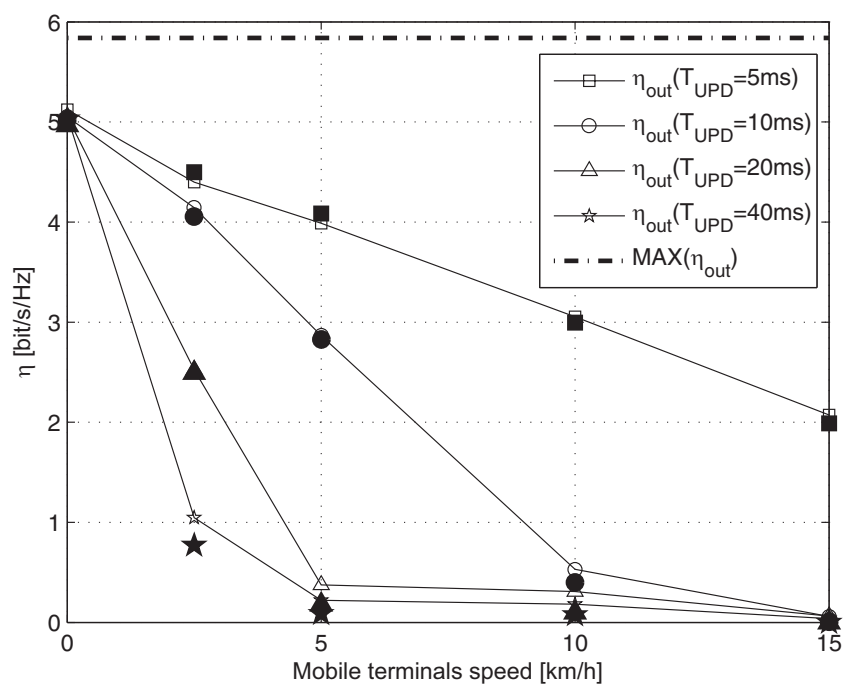

Fig. 8. Spectral efficiency $\left(\eta_{o u t}\right)$ as a function of variable mobile terminals speed $v[\mathrm{~km} / \mathrm{h}]$ with fast fading defined by pedestrian channel model Ped $-B$. Results are obtained with the analytical approach F2 (continuous lines) and compared to performance computed with intensive simulations $F 1$ (filled markers).

$T_{U P D}=40 \mathrm{~ms}$ and speed $v>5 \mathrm{~km} / \mathrm{h}$, the additional complexity introduced by the smart allocation strategy makes no sense as we can obtain the same performance with the simple CLPA or even random allocation with no power adaptation at all. In fact, the updating rate $1 / T_{U P D}$ has to be faster for making the algorithm react to the rapidly changing conditions of the wireless channel. Nevertheless, with updating time $T_{U P D}<10 \mathrm{~ms}$ (corresponding to a new resource allocation each two OFDM frames in IEEE 802.16 standard), we can see that smart algorithms are strongly recommended for achieving a satisfactory transmission rate up to velocities around $5 \mathrm{~km} / \mathrm{h}$.

Although radio resource allocation solutions have demonstrated their ability to increase the spectral efficiency of mobile users, it has still to be considered their impact on the computational complexity. In other words, it should be evaluated the level of complexity that can be supported by the processing units, giving rise to the trade-off between performance and sustainable computational complexity. In Figs. 12 and 13 we point out the relation between computational complexity and performance by using performance evaluations expressed as a function of the partitioning factor $P$. Curves with constant spectral efficiency ( $\eta_{\text {out }}=1,2,3,4,5$ $[\mathrm{bit} / \mathrm{s} / \mathrm{Hz}])$ are depicted as a function of the partitioning factor of sub-channels and users $(P)$, velocity and updating time. We can notice that the best value $\eta_{\text {out }}=5$ can be achieved only if we adopt the algorithm at the maximum complexity $(P=1)$ and with fixed users $(v=0 \mathrm{~km} / \mathrm{h})$. In general, a high level of complexity corresponds to higher levels of $\eta_{\text {out }}$ even with mobile users. However, we observe also that a complexity reduction might allow a faster updating time, which always guarantees higher performance. So, in these figures, we can appreciate the overall trade-off among computational complexity, expressed by the partitioning factor $P$, updating time and achievable $\eta_{\text {out }}$. This kind of simulation or analysis 


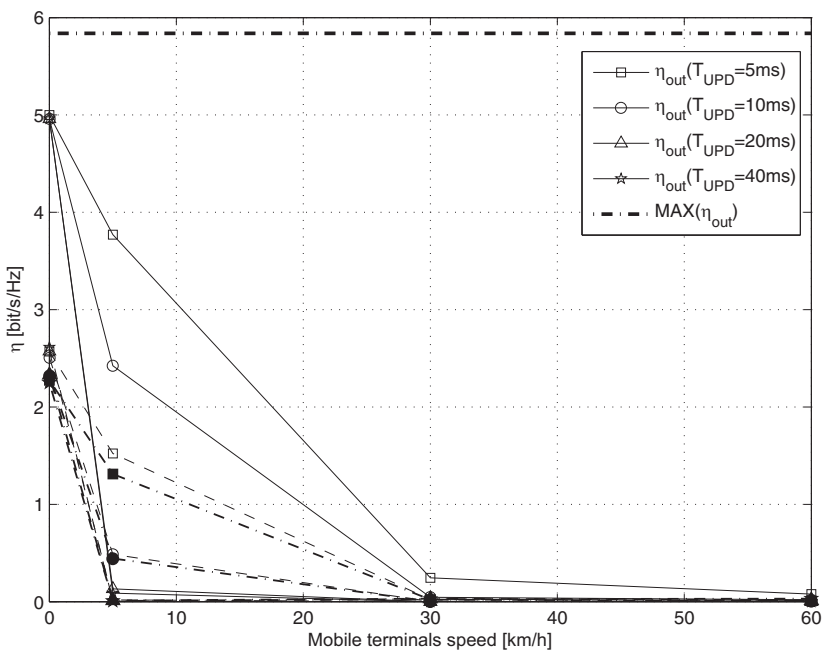

Fig. 9. Spectral efficiency $\left(\eta_{\text {out }}\right)$ as a function of users velocity $(v)$ and updating time $\left(T_{U P D}\right)$ with vehicular channel model $\mathrm{Veh}-\mathrm{A}$. Performance of radio resource allocation algorithm with $I_{D}=N_{U}$ (continuous lines ' - '), simple CLPA with $I_{D}=1$ (dashed lines '- -'), and random allocation without any power adaptation (dotted lines '-..') are compared.

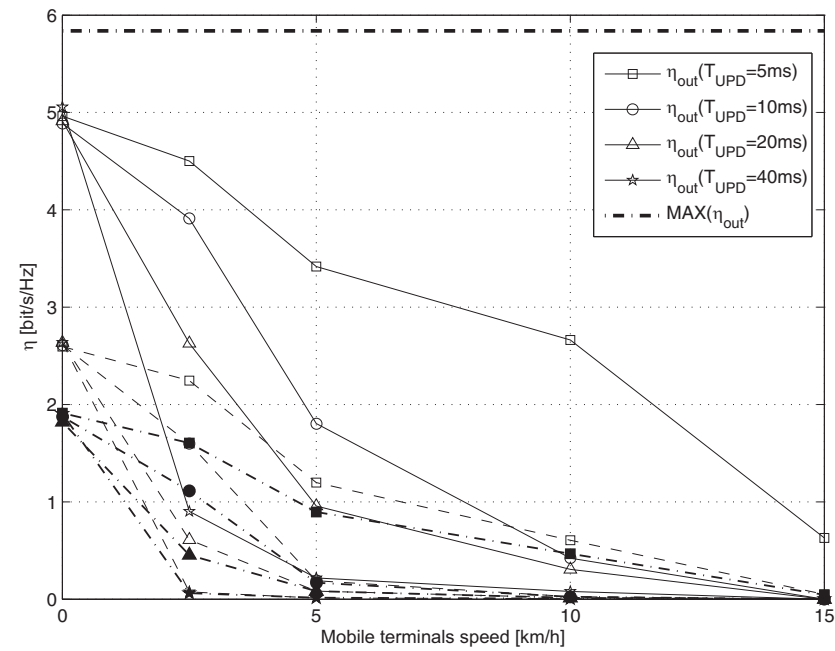

Fig. 10. Spectral efficiency $\left(\eta_{\text {out }}\right)$ as a function of users velocity $(v)$ and updating time $\left(T_{U P D}\right)$ with pedestrian channel model $\mathrm{Ped}-A$. Performance of radio resource allocation algorithm with $I_{D}=N_{U}$ (continuous lines '-'), simple CLPA with $I_{D}=1$ (dashed lines '- -'), and random allocation without any power adaptation (dotted lines '-..') are compared. 


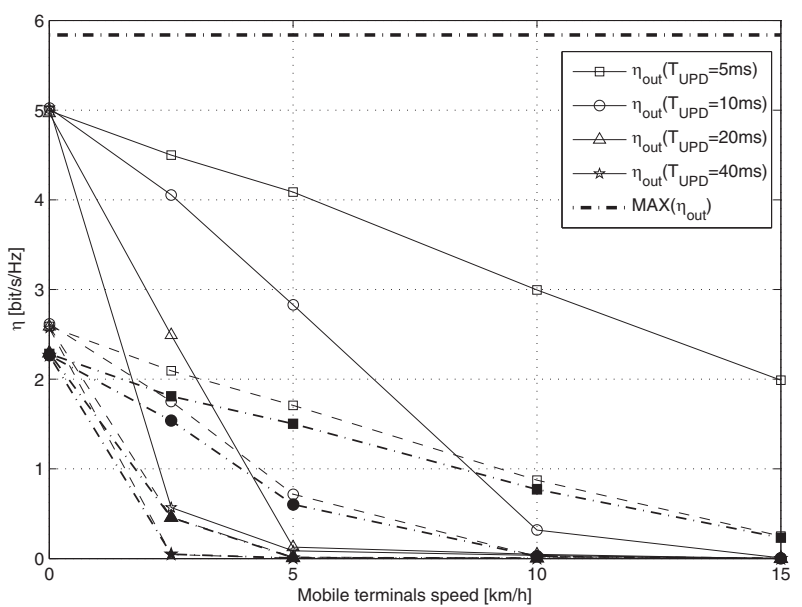

Fig. 11. Spectral efficiency $\left(\eta_{\text {out }}\right)$ as a function of users velocity $(v)$ and updating time $\left(T_{U P D}\right)$ with pedestrian channel model Ped $-B$. Performance of radio resource allocation algorithm with $I_{D}=N_{U}$ (continuous lines '-'), simple CLPA with $I_{D}=1$ (dashed lines '- -'), and random allocation without any power adaptation (dotted lines '-.-') are compared.

reveals the possible design choices that can be adopted in a multi-cellular system, according to the system updating or response time and to the BS processing power.

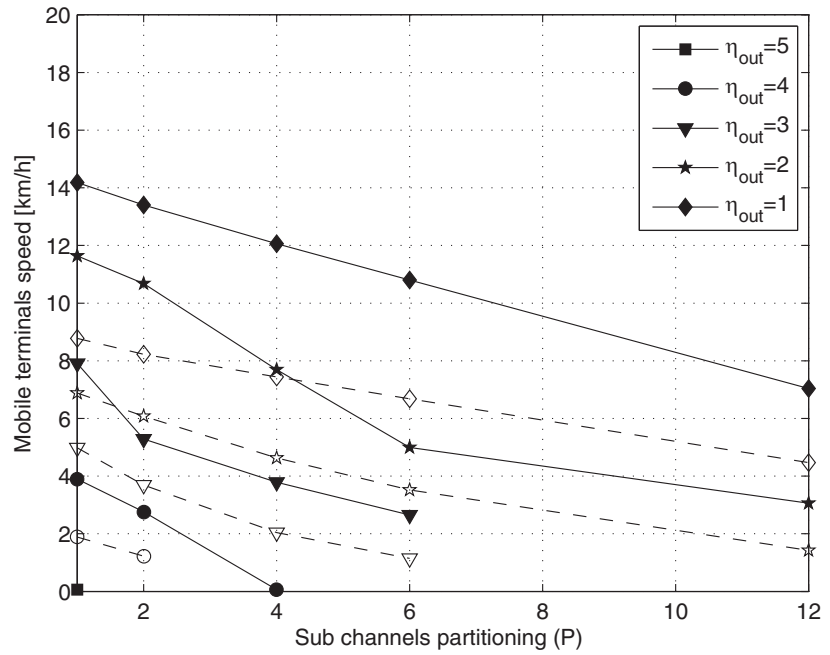

Fig. 12. Curves at fixed $\eta_{o u t}$ as a function of user velocity $(v)$, partitioning factor $(P)$ and updating time $T_{U P D}=5 \mathrm{~ms}$ (continuous line ' - ') and $T_{U P D}=10 \mathrm{~ms}$ (dashed line '- -') in the presence of pedestrian channel model Ped $-A$. 


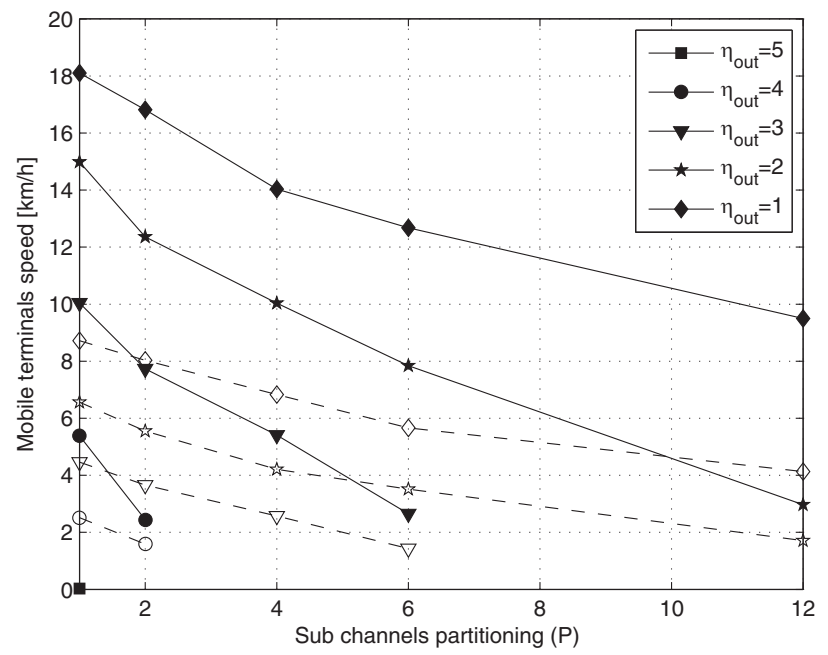

Fig. 13. Curves at fixed $\eta_{\text {out }}$ as a function of user velocity $(v)$, partitioning factor $(P)$ and updating time $T_{U P D}=5 \mathrm{~ms}$ (continuous line ' $-{ }^{\prime}$ ) and $T_{U P D}=10 \mathrm{~ms}$ (dashed line '- $\left.{ }^{\prime}\right)$ in the presence of pedestrian channel modelPed $-B$.

When mobile users are at different and varying distances $d$ from the BSs, the numerical findings confirm the same performance behavior described below. Also uplink simulations show similar results even if with lower values of $\eta_{\text {out }}$.

\section{Conclusions}

In the chapter, we have investigated the impact of allocation strategies on multi-cell networks with mobile users. The power reduction that can be achieved by means of the multi-user diversity exploitation has a beneficial impact on the overall network interference with a successive improvement of spectral efficiency. This positive effect is present, even if clearly reduced, also when only an efficient power adaptation loop, without smart allocation, is implemented in the network. On the other hand, it is shown how the users velocity has a strong impact on the updating time that is necessary for maintaining a satisfactory performance. This trade-off is completed by the algorithm complexity, which is another fundamental parameter that affects the updating ability of the system. In this context, a set partitioning technique is presented as a step for reducing the complexity order of smart allocation towards very fast algorithms that are compatible with low updating times.

\section{References}

Knop, R. \& Humblet, P. (1995). Information Capacity and Power Control in Single-cell Multiuser Communications. Proceedings of IEEE ICC 1995, June 1995, Seattle (WA, USA)

Galati Giordano, L. \& Reggiani, L. \& Dossi, L. (2010). Radio Resource Management for New Generation Wireless Networks: Smart Allocation Techniques and Interference Evaluation. VDM Verlag Dr. Müller, ISBN 978-3-639-26548-4, 112 pages, June 2010 
Reggiani, L. \& Galati Giordano, L. \& Dossi, L. (2007). Multi-User Sub-Channel, Bit and Power Allocation In IEEE 802.16 systems. Proceedings of IEEE VTC-2007 Spring, April 2007

Galati Giordano, L. \& Reggiani, L. \& Dossi, L. (2008). Interferente Evaluation in Multi-Cell Environment with Resource Allocation Algorithms. Proceedings of IEEE VTC-2008 Spring, May 2008

IEEE Std 802.16-2004. IEEE Standard for Local and Metropolitan Area Networks: Air Interface for Fixed Broadband Wireless Access Systems, October 2004

IEEE Std 802.16e-2005 \& IEEE Std 802.16-2004/Cor1-2005. IEEE Standard for Local and Metropolitan Area Networks IEEE: Air Interface for Fixed and Mobile Broadband Wireless Access Systems and Corrigendum 1, February 2006

3GPP TR 25.996. Universal Mobile Telecommunication System (UMTS): Spatial Channel Model for Multiple Input Multiple Output (MIMO) Simulations, Release 6, September 2003

ETSI TR 101112 (1998-04). Selection Procedures for the Choice of Radio Transmission Technologies of the UMTS Ü Annex B: Test Environments and deployment models, UMTS 30.03, version 3.2.0, 1998

Schoenen, R. \& Qin, F. (2009). Adaptive Power Control for 4G OFDMA systems on Frequency Selective Fading Channels. Proceedings of WiCOM 2009, March 2009

Li, Z. \& Wang, Y. \& Yang, D. (2008). A Novel Power Control Scheme in OFDMA Uplink. Proceedings of ICSP 2008, May 2008, Leipzig (Germany)

Tee, L.K. \& van Rensburg, C. \& Tsai, J.-A. (2007). Uplink Power Control for an OFDMA Mobile Cellular System. Proceedings of IEEE VTC-2007 Fall, September 2007, Baltimore (MD, USA) 


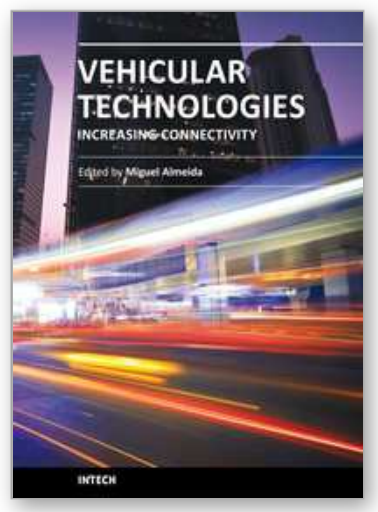

\author{
Vehicular Technologies: Increasing Connectivity \\ Edited by Dr Miguel Almeida
}

ISBN 978-953-307-223-4

Hard cover, 448 pages

Publisher InTech

Published online 11, April, 2011

Published in print edition April, 2011

This book provides an insight on both the challenges and the technological solutions of several approaches, which allow connecting vehicles between each other and with the network. It underlines the trends on networking capabilities and their issues, further focusing on the MAC and Physical layer challenges. Ranging from the advances on radio access technologies to intelligent mechanisms deployed to enhance cooperative communications, cognitive radio and multiple antenna systems have been given particular highlight.

\title{
How to reference
}

In order to correctly reference this scholarly work, feel free to copy and paste the following:

L. Reggiani, L. Galati Giordano and L. Dossi (2011). Fast Power and Channel Adaptation for Mobile Users in OFDMA Multi-Cell Scenarios, Vehicular Technologies: Increasing Connectivity, Dr Miguel Almeida (Ed.), ISBN: 978-953-307-223-4, InTech, Available from: http://www.intechopen.com/books/vehicular-technologiesincreasing-connectivity/fast-power-and-channel-adaptation-for-mobile-users-in-ofdma-multi-cell-scenarios

\section{INTECH}

open science | open minds

\section{InTech Europe}

University Campus STeP Ri

Slavka Krautzeka 83/A

51000 Rijeka, Croatia

Phone: +385 (51) 770447

Fax: +385 (51) 686166

www.intechopen.com

\section{InTech China}

Unit 405, Office Block, Hotel Equatorial Shanghai

No.65, Yan An Road (West), Shanghai, 200040, China

中国上海市延安西路65号上海国际贵都大饭店办公楼 405 单元

Phone: +86-21-62489820

Fax: +86-21-62489821 
(C) 2011 The Author(s). Licensee IntechOpen. This chapter is distributed under the terms of the Creative Commons Attribution-NonCommercialShareAlike-3.0 License, which permits use, distribution and reproduction for non-commercial purposes, provided the original is properly cited and derivative works building on this content are distributed under the same license. 